\title{
Topographical and Physicochemical Contrast in Photopatterned SU-8 Films for Microfabrication of Multilayer Structures
}

\author{
L. Salazar Alarcón, ${ }^{1}$ E. D. Martínez, ${ }^{1}$ L. M. Rodríguez, ${ }^{2}$ and H. Pastoriza ${ }^{1}$ \\ ${ }^{1}$ Laboratorio de Bajas Temperaturas-Instituto de Nanociencia y Nanotecnología (INN), Centro Atómico Bariloche, \\ Comisión Nacional de Energía Atómica, CONICET, Avenida Bustillo 9500, R8402AGP S.C. Bariloche, 8400 Rio Negro, Argentina \\ ${ }^{2}$ Laboratorio de Colisiones Atómicas, Centro Atómico Bariloche, Comisión Nacional de Energía Atómica, CONICET, \\ Avenida Bustillo 9500, R8402AGP S.C. Bariloche, 8400 Rio Negro, Argentina
}

Correspondence should be addressed to L. Salazar Alarcón; ldsalazaralarcon@gmail.com

Received 29 February 2016; Revised 25 April 2016; Accepted 3 May 2016

Academic Editor: Mikhael Bechelany

Copyright (C) 2016 L. Salazar Alarcón et al. This is an open access article distributed under the Creative Commons Attribution License, which permits unrestricted use, distribution, and reproduction in any medium, provided the original work is properly cited.

\begin{abstract}
During SU-8 standard photolithography process, a patterned topography is formed with a characteristic height profile produced by the different shrinkage of the UV exposed and masked regions. We study the change of wettability, film solubility, and topographic modifications on SU-8 films of different thicknesses and show its relevance in the formation of spinning-flow arrays on top layers made from positive photoresists. Also, considerable contrast in film solubility and surface energy as observed from contact angle measurements is produced. Interface diffusion of the photoresists was also observed and followed by Rutherford Back Scattering. We discuss the derivations of the mentioned effects concerning the limitations to multilayered microfabrication processes and possibilities to take advantage of the surface profiles obtained.
\end{abstract}

\section{Introduction}

Polymeric photoresists are employed in microfabrication as standard materials for photolithography. However, photoresists are typically employed as sacrificial materials that only serve to transfer designed features on a substrate. Other materials, such as metals, oxides, and semiconductors, are used to conform to the devices envisioned. In recent years, new processes have emerged that make use of polymers as the functional and structural materials. A growing number of polymer based microdevices, mainly applied in microfluidics, can be found in the literature [1-6]. The growing interest in polymer based MEMS [7] has driven the development of novel processes and protocols to control its mechanical [8-10], optical [11], magnetic [12-14], and electrical [15-17] properties. Also the surface chemistry [18], the resolution attainable during optical lithography [19], and the topographical features were intensively studied.

Because of the intrinsic mechanical properties of epoxies, SU-8, an epoxy based negative tone resist, is widely used for polymer MEMS [20-22]. However, the lithography process involves several thermal treatments and multilayer coatings, which are successively patterned to achieve the desired $3 \mathrm{D}$ structures. It is important to notice the existence of many problems during multilayer fabrication. For example, although it is commonly assumed that photoresists of uniform thickness achieve the designed pattern only after the dissolution of the exposed/unexposed regions to UV light for positive/negative tone resists, it was observed that topographical changes are produced on SU-8 before the development step. This is due to the differential shrinkage of the exposed and masked regions during the so-called postexposure bake (PEB) [23-25].

In this work, we aim to identify, classify, and resolve the main and key factors tangled with microfabrication involving bilayers of different photoresists, in search of accurate procedures for polymer MEMS fabrication. We studied the bilayer system form by a positive tone resist over a prepatterned, but undeveloped, structural layer of SU-8. We report that, besides the topographical changes, UV exposure affects two additional physicochemical properties. These are the different wettability of a secondary, top resist, over the exposed and 
TABLE 1: Conditions for deposition and lithography of each layer.

\begin{tabular}{|c|c|c|c|c|c|c|c|}
\hline & $\begin{array}{l}\text { Spin max speed } \\
\text { (RPM) }\end{array}$ & SB & PEB & HB & Exposure & $\begin{array}{c}\text { Dose } \\
\left(\mathrm{mJ} / \mathrm{cm}^{2}\right)\end{array}$ & $\begin{array}{l}\text { Development time } \\
\text { (s) }\end{array}$ \\
\hline SU-8 2001 & 3000 & $60 \mathrm{~s}$ at $65^{\circ} \mathrm{C} \& 60 \mathrm{~s}$ at $95^{\circ} \mathrm{C}$ & $S B$ & $15 \mathrm{~min}$ at $200^{\circ} \mathrm{C}$ & Mask & 162 & 60 \\
\hline SU-8 2010 & 3500 & $60 \mathrm{~s}$ at $65^{\circ} \mathrm{C} \& 180 \mathrm{~s}$ at $95^{\circ} \mathrm{C}$ & $S B$ & $15 \mathrm{~min}$ at $200^{\circ} \mathrm{C}$ & Mask & 189 & 180 \\
\hline SU-8 2050 & 4000 & $180 \mathrm{~s}$ at $65^{\circ} \mathrm{C} \& 240 \mathrm{~s}$ at $95^{\circ} \mathrm{C}$ & $S B$ & $15 \mathrm{~min}$ at $200^{\circ} \mathrm{C}$ & Mask & 324 & 300 \\
\hline AZ-9260 & 2500 & $210 \mathrm{~s}$ at $105^{\circ} \mathrm{C}$ & None & None & Flood & 128 & 210 \\
\hline Microposit S1400-31 & 5500 & $180 \mathrm{~s}$ at $95^{\circ} \mathrm{C}$ & None & None & Flood & 108 & 45 \\
\hline
\end{tabular}

unexposed regions of the SU-8 bottom layer, and the partial solubility of the SU-8, giving rise to interface diffusion and intermixing between the two layers. We recommend actions to avoid or reduce the drawbacks produced by these effects. We find these factors to be highly relevant for the fabrication of multilayer polymer based microdevices and shed light on this topic.

\section{Experimental Procedure}

Sample Preparation. Two kinds of photoresists were used for the fabrication of bilayer structures. The bottom layer was made from epoxy based SU-8 (MicroChem Corp.) as negative tone photoresists in 2001, 2010, and 2050 formulations. For the top layer positive tone resists like AZ-9260 (AZ Electronics) and Microposit S1400-31 (Shipley) were used. Photoresist films were deposited over soda-lime glass slides by spin coating and all samples were cured by soft bake (SB) as recommended by the supplier. Lithography was performed using a SÜSS Mask Aligner MJB4 with an OSRAM $350 \mathrm{~W} \mathrm{Hg}$ lamp. Between the lamp and the mask a bandpass optical filter was set for cutting off wavelengths below $350 \mathrm{~nm}$. SU-8 films require PEB after UV exposure for cross-linking of epoxy groups. An optional hard bake (HB) process at higher temperature can be optionally performed. A top layer with a positive tone resist was spin coated followed by a second SB necessary for drying. A second UV flood exposure was applied to this layer. Finally, top layer was developed using AZ 400K 1: 4 developer. The characteristic parameters employed in the process for each layer are detailed in Table 1.

Wetting Angle Measurements. We prepare SU-8 2050 films processed by three different treatments: first, SU- 8 was spin coated on glass slide and SB was performed. A sample of this film was conserved, while the rest of the slide was irradiated with $336 \mathrm{~mJ} / \mathrm{cm}^{2} \mathrm{UV}$ in flood exposure mode. Afterwards, PEB was applied and a piece of this sample was taken. The remaining piece was submitted to a hard bake for $15 \mathrm{~min}$ at $200^{\circ} \mathrm{C}$. The wetting angle was measured using a homemade setup with a generic 200x digital microscope for drop magnification; the drop has a constant volume of $2 \mu \mathrm{L}$ deposited with a micropipette. The images were analyzed to obtain the contact angle for water and Microposit on SU8 film with SB, PEB, and HB. The images were analyzed to obtain the contact angle for water and Microposit on SU-8 film submitted to SB, PEB, and HB using an image manipulation program (GIMP) [26] to extract the edge drop and measure the contact angle with the surface.
Sample Surface Topography. The topographical features produced after PEB in different formulations of SU-8 films were analyzed by AFM (Bruker microscope) in contact mode. SEM images were performed in SEM Philips XL-30 at $30 \mathrm{keV}$. Optical images were obtained with a Nikon Eclipse L200 microscope.

Top Layer Diffusion. Absorption spectra were taken using a Thermo-Spectronic Genesys 20 UV-visible spectrophotometer. The depth profile of sample elemental composition was measured using Rutherford Backscattering Spectrometry (RBS) with a NEC 5SDH, 1.7 MV Tandem Accelerator, and a NEC RC43 high vacuum analysis end station $\left(10^{-8}\right.$ Torr of base pressure). In brief, RBS consists in a monoenergetic ion beam ( $2 \mathrm{MeV}$ energy $\mathrm{He}^{+}$ions), impinging normal to the sample surface, backscattered by nuclei of the elements present in the sample, and detected afterwards by a surface barrier detector positioned $15^{\circ}$ apart from the beam line [27]. The outgoing energy of scattered projectiles depends on the nuclei mass and its energy distribution extends from a maximum, for nuclei at the sample surface, to lower energies due to the beam energy loss in the incoming and outgoing trajectories for nuclei at the bulk [28]. The measured spectra were analyzed with the SIMNRA [29] software that fits the experimental data by varying elemental concentrations, using a database of scattering cross sections.

\section{Results}

Bilayer Structure. The attempt to perform a bilayer structure by depositing a second layer of a positive tone resist over a previously exposed with a regular pattern, but nondeveloped SU-8 2050 film, resulted in particular features that seemed to be related to the characteristic geometry of the mask employed. A series of lines arising from a central point in the sample could be easily perceived, suggesting that centrifugal acceleration present during spin coating was responsible for the effect. A closer look at the samples allowed us to understand the way in which the spinning forces and the topology of the mask are related. The optical image shown in Figure 1 displays the flow of the positive tone resist during spinning, where each of the circular patterns of the bottom layer (SU-8) acts as an obstacle to the radial flow of the liquid. This could be either because of topographical changes or due to surface modification, and therefore wettability occurred after patterning the SU-8. However, we noticed that the appearance of such features depended on the time elapsing between the releasing of the positive resist and the start of the 


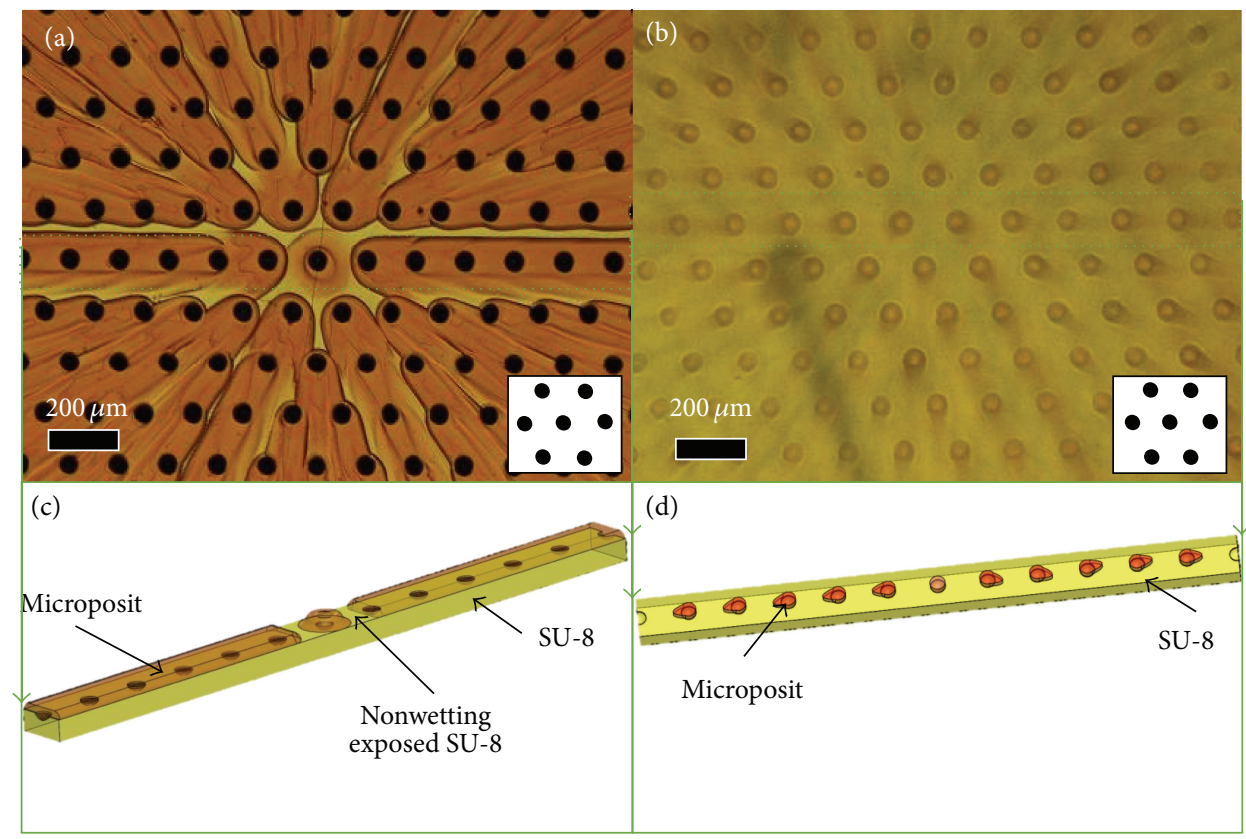

FIgURE 1: Optical images of Microposit on PEB SU-8 2050 (a) before development (b) after development. Inset shows the mask employed for UV exposure. Figures (c) and (d) represent an isometric view of the sample.

spinning cycle. Also the thickness of the SU-8 film was found to be important, since SU-8 2001 films, approximately $1 \mu \mathrm{m}$ thick, were completely deformed during the second-layer deposition. These observations suggested the presence of a third, important effect: the partial solubility of the SU-8 film in the solvent of the positive tone resists. Actually, propylene glycol monomethyl ether acetate (PGMEA, CAS: 108-65-6), which is the major solvent used in AZ-9260 and a similar compound (CAS: 111-15-9) present in Microposit resists, is also used as the major component in the recommended developer for SU-8 (SU-8 developer, MicroChem); therefore the solubility of the unexposed regions in the bottom layer and the diffusion of the positive tone resist across the SU-8 layer could be expected. A similar effect is also present when multiple layers of SU-8 are deposited.

It has to be noticed that the appearance of the radial line features is also clearly visible after the development of the top layer. After either flood or masked exposure, remaining traces of the positive colored (reddish) resists could be observed, even after extended development times. To quantify the penetration of the positive tone resist into the bottom SU-8 layer we prepared three samples of SU-8 films with SB, UV exposed plus PEB, and HB. The deposition of the Microposit resist was attempted in all of these samples. After the flood exposure of the Microposit film and its development, the characteristic reddish color of the resist was clearly visible only in the SB and PEB, but not on the HB sample. This is shown in Figure 2 where absorption spectra were taken after the development of Microposit top layer.

The result of this experiment suggested that the solubility and penetration of the positive tone resist were reduced after progressive SU-8 thermal treatment and specially restrained

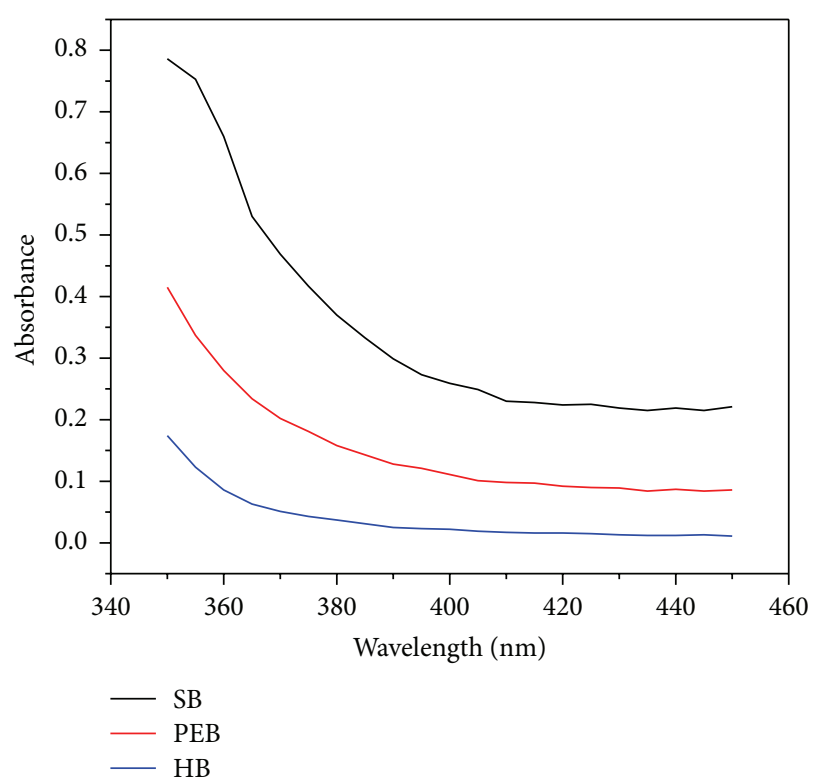

FIGURE 2: UV-visible absorption spectra of bilayer samples formed by deposition of Microposit over different thermally treated SU-8 2010 films after development of Microposit layer.

after $\mathrm{HB}$, as expected from the extended cross-linking and densification of the epoxy film.

It is worth noticing that the solubility and intermixing of both resists take place during deposition of Microposit at room temperature; however, after spinning this resist, the soft bake treatment performed at $95^{\circ} \mathrm{C}$ could also enhance the interdiffusion effect because of the glass transition temperature (Tg) of SU-8. It is known that Tg of SU-8 depends on 


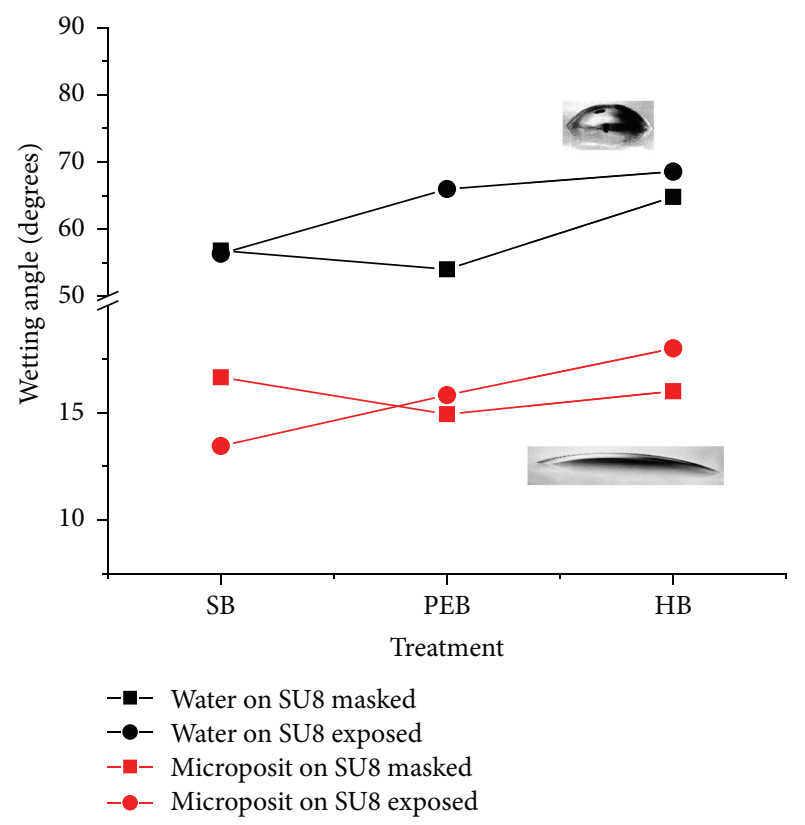

Figure 3: Contact angle for water and Microposit on SU-8 2050.

the preexposure to UV and the following thermal treatments. For the case of unexposed, soft baked SU-8, Tg is around $50^{\circ} \mathrm{C}[21,30]$; for exposed samples submitted to PEB, Tg was found to be similar to the maximum temperature during PEB $\left(95^{\circ} \mathrm{C}\right.$ in our case) (Feng). For hard baked SU-8, Tg is $>200^{\circ} \mathrm{C}[19,21,30]$. This is also in agreement with our observations, being the sample submitted to SB the one that shows the higher absorption due to remaining Microposit resist, as shown in Figure 1.

(1) Wettability. Regarding the change in wettability it was observed that masked and exposed regions of the SU-8 show different wetting properties for water and Microposit positive tone resist. Wetting angles were measured. The results for SU-8 2050 are summarized in Figure 3 where an increase in the contact angle can be noticed after each of the thermal treatments successively applied. This means a reduction of wettability for Microposit due to the thermal treatment of SU-8. Microposit resist displays better wettability compared to water. Wetting angles for water can be compared with typical values reported in the literature $\left(60^{\circ}-80^{\circ}\right)$ [31-35]. Water wettability is also important because the majority of developers products for positive tone resist are aqueous based solutions. Our results show that exposed regions of the SU8 film are more hydrophobic, in agreement with previous studies.

After PEB treatment of the bottom SU-8 layer, the regions exposed have slightly less wettability than the unexposed ones. This is an initial approach to understand the reason why uncovered regions in the bottom layer are observed after spinning of Microposit. In all cases the wetting angle is higher when SU-8 is submitted to HB treatment. This differential behavior represents one of the three characteristics to take into account in the construction of bilayer structures. For SB
TABLE 2: Depth profile after PEB for different SU-8 film thicknesses.

\begin{tabular}{lccc}
\hline SU-8 & 2001 & 2010 & 2050 \\
Thickness $(\mu \mathrm{m})$ & 2 & 9 & 40 \\
Depth $(\mathrm{nm})$ & 180 & 450 & 4100 \\
Ridges height $(\mathrm{nm})$ & 10 & 100 & 420 \\
\hline
\end{tabular}

treatment the wetting behavior for Microposit is affected by partial solubility of the bottom layer as will be discussed later.

(2) Topography. The second relevant issue is the surface profile that is produced due to the differential expansion and contraction of the exposed and masked regions of SU-8 during PEB. As explained by Denning et al. [23] the cross-linking produced in the exposed regions of SU-8 films during the PEB treatment increases significantly the mechanical stiffness of the film, while the unexposed regions remain relatively softer. Both the exposed and masked regions of SU-8 films expand during the $\mathrm{PEB}$ at $95^{\circ} \mathrm{C}$; however, due to the cross-linking in the exposed regions, it becomes more rigid [36] and during cooling contracts less than the masked regions, creating then a topographical profile where unexposed regions are seen as depressed features, surrounded by ridges formed in the frontier between both areas. In the case studied by Feng and Farris [36], the SU-8 film thickness was approximately $15 \mu \mathrm{m}$ and the valleys measured were about $400 \mathrm{~nm}$, while the heights of the ridges were nearly $200 \mathrm{~nm}$.

We studied this effect in detail by using a transparent mask with $50 \mu \mathrm{m}$ opaque disks arranged in hexagonal pattern. After PEB, AFM scans were performed to quantify the topographical features and also by means of SEM imaging of the sample placed at a tilted angle. Both results are shown in Figure 4. It was confirmed that the topographical features are more pronounced in thicker films (Table 2) since the absolute volume changes are more significant.

The third effect relevant to bilayer fabrication analyzed in this work is the solubility of bottom layer during the coverage with top layer.

(3) Interface Diffusion. To better understand the effects such as partial diffusion of top layer into the bottom one, we used RBS to follow chemistry markers contained in one of the layers involved, for example, antimony (Sb) and sulfur (S) that are present in the SU-8 photoinitiator (triarylsulfonium hexafluoroantimonate salts) but are not in the Microposit photoresists.

Figure 5 shows RBS spectra for SU-8 2010 with different treatment stages and a second Microposit photoresist layer equally baked, exposed, and developed for all samples. Although the total film thickness is around $10 \mu \mathrm{m}$, the penetration depth of the beam is $5 \mu \mathrm{m}$. In this region of the sample, steps corresponding to every element can be observed. The split shoulder structure can be assigned to $\mathrm{C}$, $\mathrm{O}$, and $\mathrm{S}$ elements. The peak observed at $1350 \mathrm{keV}$ indicates that there is a thin region of the film containing potassium. Besides the above-mentioned elements there is an estimated amount of $85 \%$ atoms of $\mathrm{H}$ (invisible by this technique). Elements such as $\mathrm{C}$ and $\mathrm{O}$ are present in SU-8 as well as 


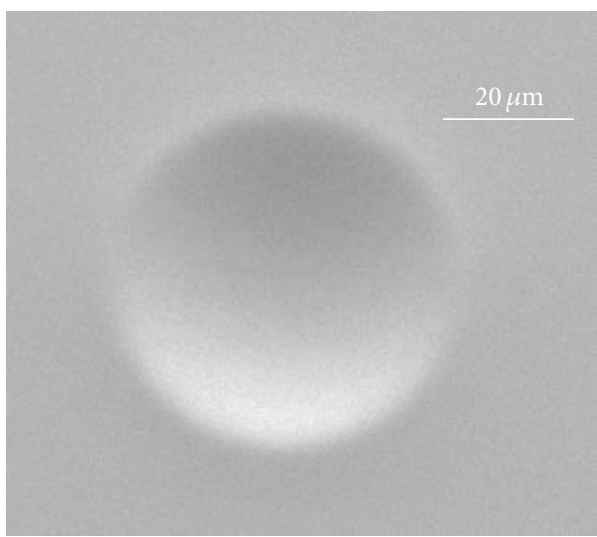

(a)

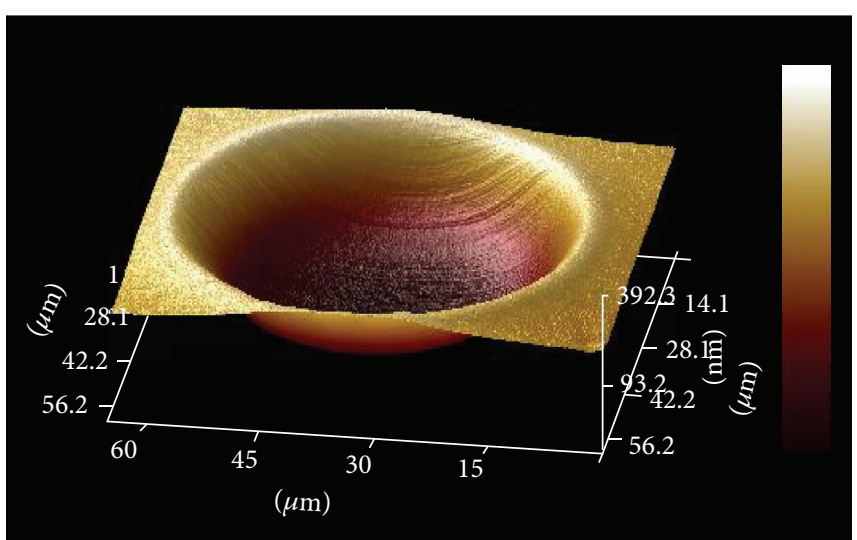

(b)

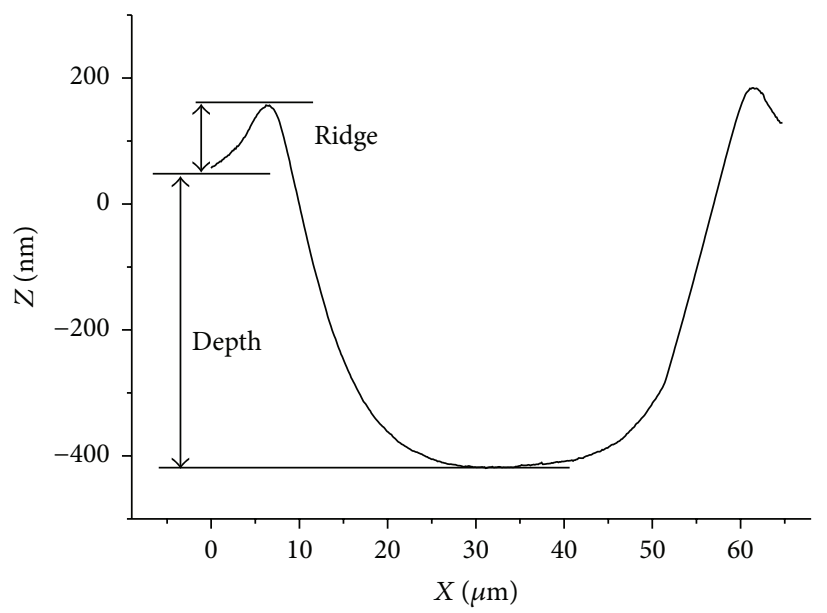

(c)

FIGURE 4: $50 \mu \mathrm{m}$ circle in SU-8 2010 after PEB: (a) SEM photograph. (b) AFM. (c) AFM profile.

Microposit while $\mathrm{K}$ detected is a residue from the developer solution.

SB and PEB samples seem to form a mixture of both resists on top, contaminated with $\mathrm{K}$ belonging to developer, keeping the unchanged bottom of SU-8 out of the reach of the beam. An interdiffusion layer is formed due to partial dilution of SU-8 layer. Sulfur and epoxy groups migrate into this mixture layer. The composition of the mixture layer is slightly enriched with S, having $1.15 \%$ for PEB and $0.82 \%$ for SB.

PEB sample shows a more notorious interdiffusion effect, contrary to the expectation to get a denser structure after cross-linking produced during PEB. In this case, the S step suggests also a variation in the composition from the surface to the bulk, explained by the deviation from the simulated plateau between 800 and $1300 \mathrm{keV}$. The slope states that the concentration of $\mathrm{S}$ is higher in deeper regions of the sample approaching that of pure SU-8.

Sulfur content in $\mathrm{HB}$ sample was notoriously lower $(0.18 \%)$ possibly due to thermal decomposition of sulfur based photoinitiator molecule as reported by Takahashi and Shirai [37] who found this effect starting at $150^{\circ} \mathrm{C}$.
In case of $\mathrm{PEB}$, the thicker interdiffusion region compares to penetration depth, making Sb out of reach. This seems reasonable to us as a result of interdiffusion phenomena between resist compounds because the diffusion coefficient for $S$ is higher than for Sb. SB and HB samples spectra have a clear Sb step at highest scattering energy.

\section{Conclusions}

Three different effects were found to be important for the microfabrication of multilayer structures based in SU-8 resist: the differential wettability of the exposed and masked regions of the SU-8 films, the topographical features arising during $\mathrm{PEB}$, and the solubility and interdiffusion of positive tone resists and SU-8 films. The mentioned issues affect significantly the quality of the bilayer photolithography and the resolution attainable and limit then the performance of designed devices. We find that several actions could be taken to minimize the importance of these effects such as choosing thinner SU-8 films, reducing the resting time of the positive resist before starting the spinning cycle, and performing a hard bake, whenever possible, of the SU-8 film before the 


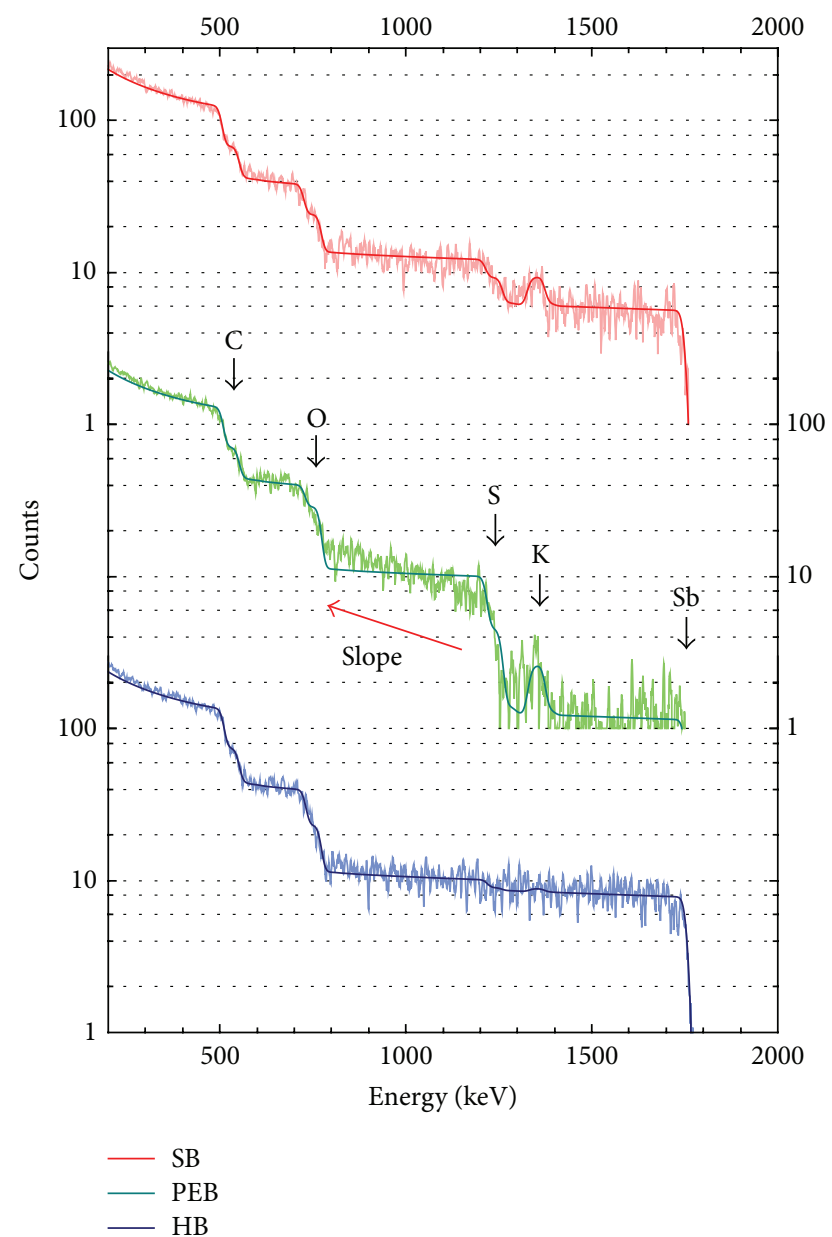

FIGURE 5: RBS spectra for Microposit/SU-8 samples with different SU-8 2010 treatments: SB (red), PEB (green), HB (blue), and SIMNRA software fittings (dark color lines).

deposition of the top layer. RBS spectra confirm that with the HB SU-8 process it is possible to get two well defined layers as interface diffusion effects occur when SU-8 is not hard baked. We called the fact that the topographical features could also provide an advantage for the design of nanostructured surfaces and molds for soft-lithography techniques, where controlled roughness and curved profiles as shown in Figure 3 could be useful.

\section{Competing Interests}

The authors declare that there is no conflict of interests regarding the publication of this paper.

\section{Acknowledgments}

Special thanks to go Martín Sirena and Luis Avilés from Bariloche's Magnetic Resonance Laboratory Group for AFM measurements, Daniel Fregenal from Atomic Collisions Laboratory Group for RBS measurements, and Claudia Osuna from INVAP for UV-visible measurements. The authors like to acknowledge the financial support of CONICET and ANPCYT (PICT 2012/770).

\section{References}

[1] I. G. Foulds and M. Parameswaran, "A planar self-sacrificial multilayer SU-8-based MEMS process utilizing a UV-blocking layer for the creation of freely moving parts," Journal of Micromechanics and Microengineering, vol. 16, no. 10, article 026, pp. 2109-2115, 2006.

[2] S. L. Tao and T. A. Desai, "Microfabrication of multilayer, asymmetric, polymeric devices for drug delivery," Advanced Materials, vol. 17, no. 13, pp. 1625-1630, 2005.

[3] H. Zou, J. Li, P. Jurčíček, and G. Wang, "Microfabrication of a multilayer nano-ESI focusing electrode based on SU-8 material," Microelectronic Engineering, vol. 103, pp. 150-155, 2013.

[4] A. P. Gadre, A. J. Nijdam, J. A. Garra et al., "Fabrication of a fluid encapsulated dermal patch using multilayered SU-8," Sensors and Actuators, A: Physical, vol. 114, no. 2-3, pp. 478-485, 2004.

[5] B. Bohl, R. Steger, R. Zengerle, and P. Koltay, "Multi-layer SU-8 lift-off technology for microfluidic devices," Journal of Micromechanics and Microengineering, vol. 15, no. 6, pp. 11251130, 2005.

[6] C. Fu, C. Hung, and H. Huang, "A novel and simple fabrication method of embedded SU-8 micro channels by direct UV lithography," Journal of Physics: Conference Series, vol. 34, no. 1, pp. 330-335, 2006.

[7] C. Liu, "Recent developments in polymer MEMS," Advanced Materials, vol. 19, no. 22, pp. 3783-3790, 2007.

[8] T. Morikaku, Y. Kaibara, M. Inoue et al., "Influences of pretreatment and hard baking on the mechanical reliability of SU-8 microstructures," Journal of Micromechanics and Microengineering, vol. 23, no. 10, Article ID 105016, 2013.

[9] S. Chung and S. Park, "Effects of temperature on mechanical properties of SU-8 photoresist material," Journal of Mechanical Science and Technology, vol. 27, no. 9, pp. 2701-2707, 2013.

[10] M. Mionić, S. Jiguet, M. Judelewicz, A. Karimi, L. Forró, and A. Magrez, "Study of the mechanical response of carbon nanotubes-SU8 composites by nanoindentation," Physica Status Solidi (B), vol. 247, no. 11-12, pp. 3072-3075, 2010.

[11] X.-B. Wang, J. Sun, C.-M. Chen, X.-Q. Sun, F. Wang, and D.-M. Zhang, "Thermal UV treatment on SU-8 polymer for integrated optics," Optical Materials Express, vol. 4, no. 3, pp. 509-517, 2014.

[12] M. Suter, O. Ergeneman, J. Zürcher et al., "Superparamagnetic photocurable nanocomposite for the fabrication of microcantilevers," Journal of Micromechanics and Microengineering, vol. 21, no. 2, Article ID 025023, 2011.

[13] K. Kobayashi and K. Ikuta, "Three-dimensional magnetic microstructures fabricated by microstereolithography," Applied Physics Letters, vol. 92, no. 26, Article ID 262505, 2008.

[14] N. Damean, B. A. Parviz, J. N. Lee, T. Odom, and G. M. Whitesides, "Composite ferromagnetic photoresist for the fabrication of microelectromechanical systems," Journal of Micromechanics and Microengineering, vol. 15, no. 1, pp. 29-34, 2005.

[15] M. Kandpal, C. Sharan, P. Poddar, K. Prashanthi, P. R. Apte, and V. Ramgopal Rao, "Photopatternable nano-composite (SU-8/ $\mathrm{ZnO}$ ) thin films for piezo-electric applications," Applied Physics Letters, vol. 101, no. 10, Article ID 104102, 2012.

[16] H. Cong, L. Hong, R. S. Harake, and T. Pan, "CNT-based photopatternable nanocomposites with high electrical conductivity and optical transparency," Journal of Micromechanics and Microengineering, vol. 20, no. 2, Article ID 025002, 2010. 
[17] S. Jiguet, A. Bertsch, H. Hofmann, and P. Renaud, "SU8-silver photosensitive nanocomposite," Advanced Engineering Materials, vol. 6, no. 9, pp. 719-723, 2004.

[18] J. Zhao, D. A. Sheadel, and W. Xue, "Surface treatment of polymers for the fabrication of all-polymer MEMS devices," Sensors and Actuators A: Physical, vol. 187, pp. 43-49, 2012.

[19] N. C. LaBianca and J. D. Gelorme, "Highaspect-ratio resist for thick-film applications," in Advances in Resist Technology and Processing XII, 846, vol. 2438 of Proceedings of SPIE, June 1995.

[20] R. Martinez-Duarte and M. J. Madou, "SU-8 photolithography and its impact on microfluidics," in Microfluidics and Nanofluidics Handbook, pp. 231-268, CRC Press, 2011.

[21] A. del Campo and E. Arzt, "Fabrication approaches for generating complex micro- and nanopatterns on polymeric surfaces," Chemical Reviews, vol. 108, no. 3, pp. 911-945, 2008.

[22] A. del Campo and C. Greiner, "SU-8: a photoresist for highaspect-ratio and 3D submicron lithography," Journal of Micromechanics and Microengineering, vol. 17, no. 6, pp. R81-R95, 2007.

[23] R. G. Denning, C. F. Blanford, H. Urban, H. Bharaj, D. N. Sharp, and A. J. Turberfield, "The control of shrinkage and thermal instability in SU-8 photoresists for holographic lithography," Advanced Functional Materials, vol. 21, no. 9, pp. 1593-1601, 2011.

[24] Q. Sun, K. Ueno, and H. Misawa, "In situ investigation of the shrinkage of photopolymerized micro/nanostructures: the effect of the drying process," Optics Letters, vol. 37, no. 4, pp. 710-712, 2012.

[25] L. J. Guerin, M. Bossel, M. Demierre, S. Calmes, and P. Renaud, "Simple and low cost fabrication of embedded micro-channels by using a new thick-film photoplastic," in Proceedings of the International Conference on Solid-State Sensors and Actuators (Transducers '97), vol. 2, pp. 1419-1422, June 1997.

[26] GNU, GNU Image Manipulation Program, http://www.gimp .org/.

[27] L. Provenzano, L. M. Rodríguez, D. Fregenal et al., "Measuring the stopping power of $\alpha$ particles in compact bone for BNCT," Journal of Physics: Conference Series, vol. 583, Article ID 012047, 2015.

[28] A. T. Feldman and J. Mayer, Fundamentals of Nanoscale Film Analysis, Springer, 2007.

[29] M. Mayer, "SIMNRA user's guide. Report IPP, Max-PlanckInstitut für Plasmaphysik," Tech. Rep. 9-113, 1997.

[30] R. Feng and R. J. Farris, "Influence of processing conditions on the thermal and mechanical properties of SU8 negative photoresist coatings," Journal of Micromechanics and Microengineering, vol. 13, no. 1, pp. 80-88, 2003.

[31] B. M. L. Koch, A. Amirfazli, and J. A. W. Elliott, "Wetting of rough surfaces by a low surface tension liquid," The Journal of Physical Chemistry C, vol. 118, no. 41, pp. 23777-23782, 2014.

[32] F. Walther, T. Drobek, A. M. Gigler et al., "Surface hydrophilization of SU-8 by plasma and wet chemical processes," Surface and Interface Analysis, vol. 42, no. 12-13, pp. 1735-1744, 2010.

[33] C. A. E. Hamlett, G. McHale, and M. I. Newton, "Lithographically fabricated SU8 composite structures for wettability control," Surface and Coatings Technology, vol. 240, pp. 179-183, 2014.

[34] V. Jokinen, P. Suvanto, and S. Franssila, "Oxygen and nitrogen plasma hydrophilization and hydrophobic recovery of polymers," Biomicrofluidics, vol. 6, no. 1, Article ID 016501, 2012.

[35] F. Walther, P. Davydovskaya, S. Zürcher et al., "Stability of the hydrophilic behavior of oxygen plasma activated SU-8," Journal of Micromechanics and Microengineering, vol. 17, no. 3, pp. 524531, 2007.

[36] R. Feng and R. J. Farris, "The characterization of thermal and elastic constants for an epoxy photoresist SU8 coating," Journal of Materials Science, vol. 37, no. 22, pp. 4793-4799, 2002.

[37] E. Takahashi and A. Shirai, "Novel sulfonium salts and their properties," Journal of Photopolymer Science and Technology, vol. 12, no. 1, pp. 147-152, 1999. 

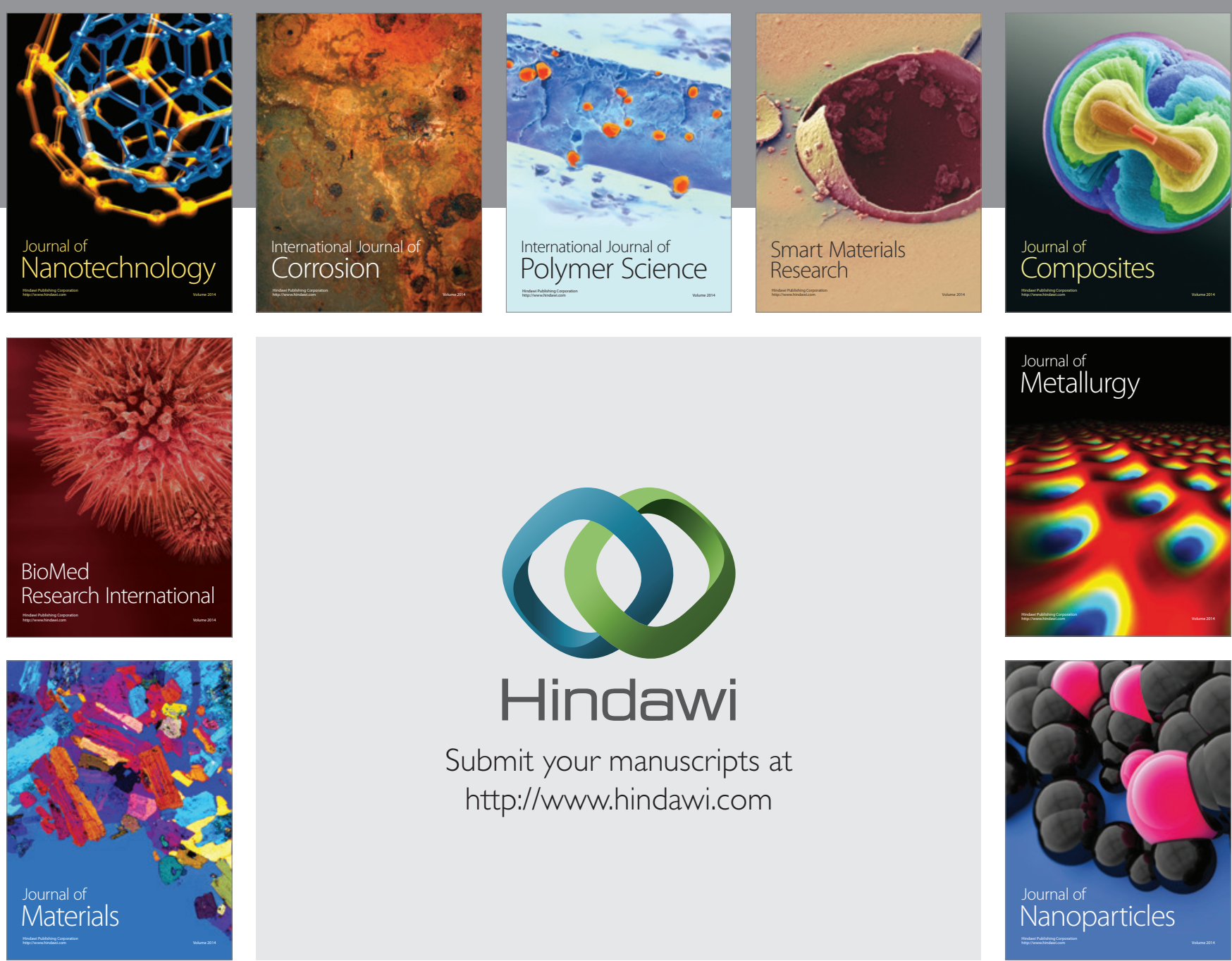

\section{Hindawi}

Submit your manuscripts at

http://www.hindawi.com

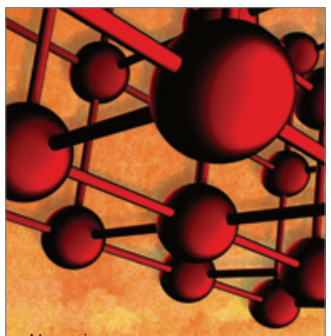

Materials Science and Engineering
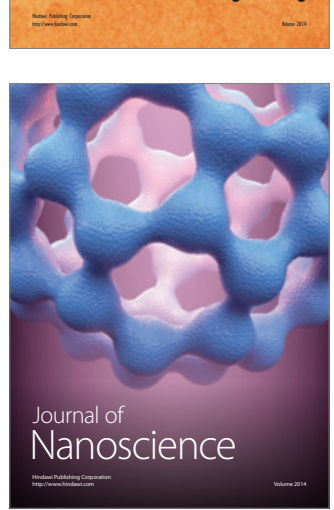
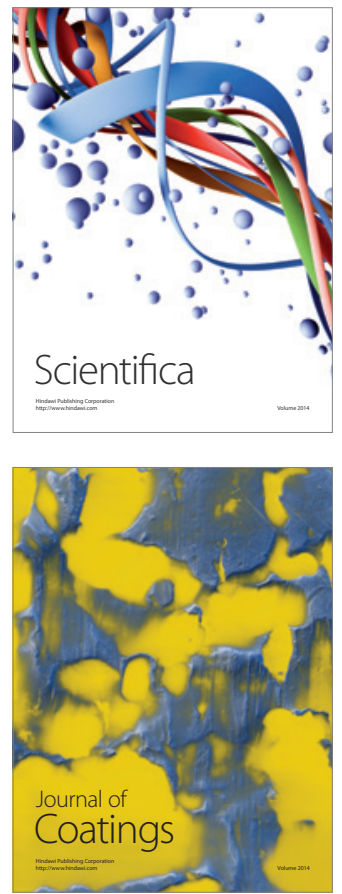
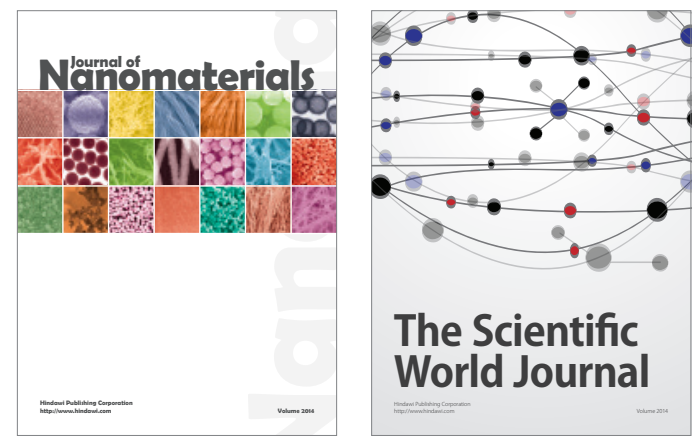

The Scientific World Journal
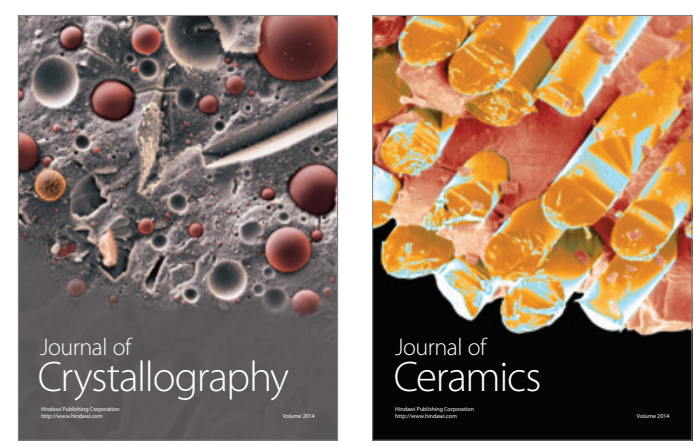
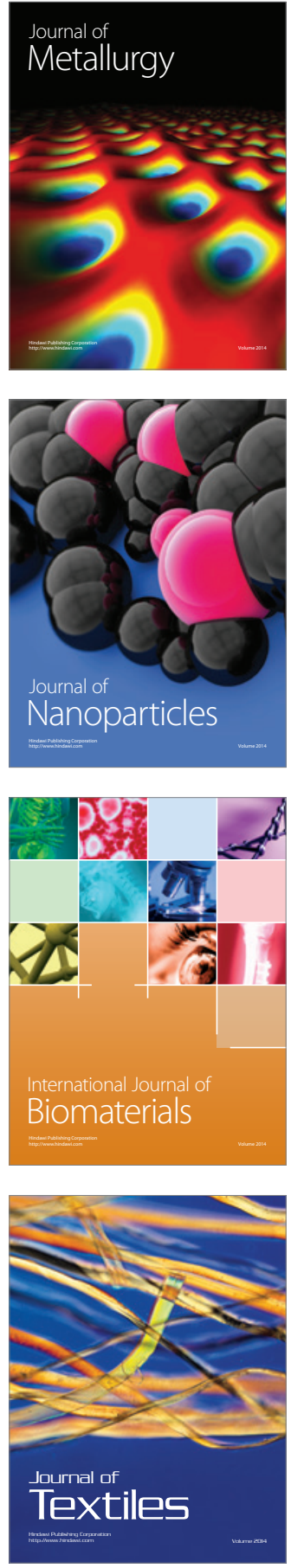